\title{
Aktuelle Behandlungskonzepte ANCA-assoziierter Vaskulitiden
}

\author{
ANCA-Associated Vasculitides: Current Therapeutic Trends
}

\section{Autor}

Institut

\section{R. Birck}

V. Medizinische Klinik, Universitätsklinikum Mannheim, Fakultät für Medizin Mannheim der Universität Heidelberg

\section{Bibliografie}

Dol $10.1055 / \mathrm{s}-2008-1077764$

Online-Publikation: 26. 11. 2008

Akt Dermatol 2009; 35:

15-18 (c) Georg Thieme

Verlag KG Stuttgart · New York

ISSN 0340-2541

Korrespondenzadresse

\section{Priv.-Doz. Dr. Rainer Birck,}

M.A.

V. Medizinische Klinik

Universitätsklinikum Mannheim

Fakultät für Medizin Mannheim der Universität Heidelberg

Theodor-Kutzer-Ufer 1-3

68169 Mannheim

rainer.birck@med5.ma.

uni-heidelberg.de

\section{Zusammenfassung \\ $\nabla$}

Die ANCA-assoziierten Vaskulitiden (AASV) zählen zu den primär systemischen Vaskulitiden mit vorwiegendem Befall kleiner und mittelgroßer Gefäße. Pathohistologisch findet sich bei allen eine nekrotisierende pauci-immune Vaskulitis, während in der Zirkulation bei der Mehrzahl der Patienten Autoantikörper gegen zytoplasmatische Bestandteile von Neutrophilen (ANCA) nachweisbar sind. Klinisch handelt es sich bei den AASV typischerweise um mehrphasisch verlaufende entzündliche Systemerkrankungen mit

\section{Einleitung \\ $\nabla$}

Als Vaskulitis werden entzündlich-nekrotisierende Veränderungen der Blutgefäße bezeichnet, die als primäre idiopathische Gefäßerkrankungen in Erscheinung treten oder sich sekundär als Gefäßmitbeteiligung einer anderen ursächlich zugrunde liegenden Erkrankung (z.B. Infektionen, Kollagenosen, Hypersensitivitätsreaktionen) manifestieren [1]. Bei den primären systemischen Vaskulitiden erfolgt heutzutage nach der Chapel-Hill-Konsensus-Konferenz von 1994 die nosologische Einteilung nach der Größe der vornehmlich vom entzündlichen Erkrankungsgeschehen befallenen Gefäße unter zusätzlicher Berücksichtung histologischer Entzündungscharakteristika, wodurch sich bestimmte distinkte klinische Entitäten voneinander abgrenzen lassen werden (siehe $\bullet$ Tab. 1) [2].

Die ANCA-assoziierten systemischen Vaskulitiden (AASV) befallen vorwiegend die kleinen bis mittelgroßen Gefäße [2]. Sie sind die am häufigsten vorkommenden primären Vaskulitiden der kleinen Gefäße des Erwachsenen [3]. Zu ihnen zählen die Wegener-Granulomatose (WG), die mikroskopische Polyangiitis (MPA) und das Churg-Strauss-Syndrom (CSS) (siehe auch häufig begleitendem rheumatischen Beschwerdekomplex. Grundsätzlich kann so gut wie jedes Organ befallen werden, wobei eine besondere Prädilektion für die Niere und die oberen und unteren Luftwege besteht. Eine Hautbeteiligung findet sich in ca. 50\% der Fälle. Moderne stadienund schweregradadaptierte immunsuppressive Therapieprotokolle erlauben eine ca. 90\% Remissionsrate, wobei wegen der chronisch-rezidivierenden Natur der AASV grundsätzlich eine anschließende Erhaltungstherapie notwendig ist. Die komplexe Behandlung der AASV sollte in hierfür spezialisierten Zentren erfolgen.

- Tab. 1) [2]. Die Inzidenz dieser Erkrankungen liegt je nach Entität bei 2 bis 10 Fällen pro 1 Million Einwohner [3]. Bei allen liegt pathohistologisch eine leukozytoklastische, fokal-nekrotisierende Vaskulitis vor. Es finden sich jedoch sowohl morphologisch in Bezug auf die entzündliche Umgebungsreaktion des betroffenen Gewebes als auch klinisch deutliche Unterschiede (siehe Tab. 2) [2,3].

Die AASV sind weiterhin durch den Nachweis zirkulierender Autoantikörper gegen Bestandteile der azurophilen Granula respektive der Lysosomen von Neutrophilen und Monozyten gekennzeichnet, den so genannten „anti-neutrophil cytoplasmic antibodies“ und werden deshalb als ANCA-assoziiert bezeichnet [2]. Die Diskrepanz zwischen diesen bei der Mehrzahl der betroffenen Patienten nachweisbaren zirkulierenden $\mathrm{Au}$ toantikörpern und den fehlenden oder nur spärlich histologisch nachzuweisenden Immunglobulinen in den betroffenen Gefäßen hat auch zu der Bezeichnung „pauci-immune Vaskulitis“ geführt [3]. Dieser Begriff, der vorwiegend seine Anwendung in der Differenzialdiagnose der rasch-progredienten Glomerulonephritiden findet [4], sollte nicht darüber hinwegtäuschen, dass sich gerade bei Hautbiopsien bei einer signi- 


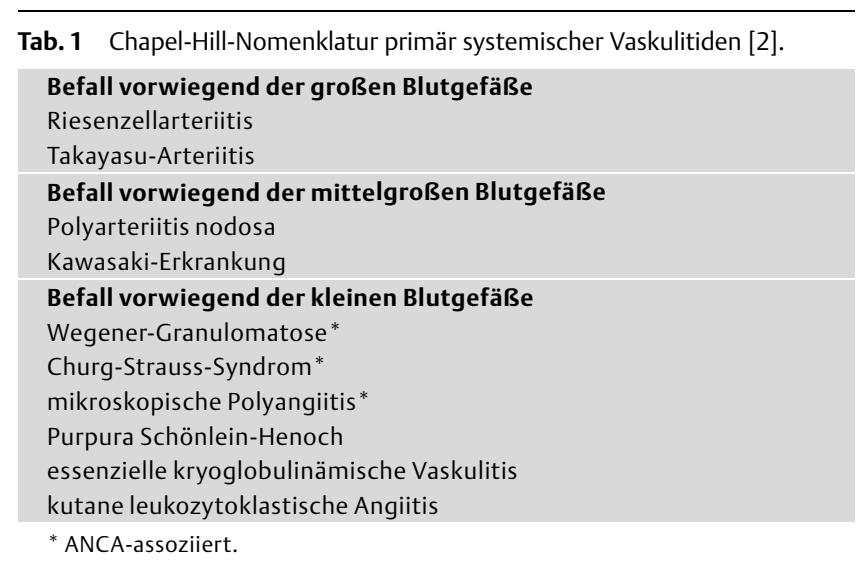

Tab. 2 Pathohistologische und klinische Charakteristika [2].

Wegener-Granulomatose
granulomatöse Entzündung des Respirationstrakts
nekrotisierende Vaskulitis der kleinen bis mittelgroßen Gefäße
Pauci-immune nekrotisierende Glomerulonephritis häufig
Mikroskopische Polyangiitis
nekrotisierende Vaskulitis kleiner und mittelgroßer Gefäße
häufig pauci-immune nekrotisierende GN
häufig pulmonale Kapillaritis
Churg-Strauss-Syndrom
eosinophilenreiche und granulomatöse Entzündung des
$\quad$ Respirationstrakts
nekrotisierende Vaskulitis der kleinen bis mittelgroßen Gefäße
Bluteosinophilie
assoziiert mit Asthma

fikanten Anzahl von Patienten relevante Immunglobulinablagerungen nachweisen lassen [5].

Klinisch handelt es sich bei den AASV typischerweise um mehrphasisch verlaufende entzündliche Systemerkrankungen mit häufig begleitendem rheumatischem Beschwerdekomplex [1]. Grundsätzlich kann durch die AASV so gut wie jedes Organ befallen werden. Bei der WG und der MPA findet sich typischerweise eine pauci-immune nekrotisierende Glomerulonephritis beziehunsgweise eine pulmonale Kapilariitis [1]. Die WG und das CSS zeigen weiterhin beide granulomatöse Entzündungen im Bereich der oberen- und unteren Luftwege [1]. Spezifisch für das CSS ist neben der immer vorliegenden Asthma-Anamnese eine ausgeprägte Blut- und Gewebseosinophilie, darüber hinaus besteht sehr häufig eine Herzbeteiligung [1]. Eine kutane Beteiligung zeigen 40-60\% der AASV-Patienten, wobei klinisch sich in der Mehrzahl eine klassische palpable Purpura an abhängigen Körperpartien findet. Es sind allerdings je nach Größe und Lage der befallenen Gefäße auch Erytheme, Hämorrhagien, subkutane Knoten, Ulcerationen oder Nekrosen als Hautbeteiligung beschrieben [6].

Vor der Etablierung des Stickstofflost-Derivats Cyclophosphamid (CYC) Anfang der 70er-Jahre war die Prognose der WG ohne Behandlung mit einer mittleren Überlebenszeit von fünf Monaten und einer Mortalität von $80 \%$ innerhalb von 12 Monaten infaust [7]. Mit der zu dieser Zeit eingesetzten Steroidmonotherapie konnte trotz der Verwendung hoher Dosierungen und zu beobachtender kurzfristiger Verbesserungen nur eine unbefriedigende Verlängerung des mittleren Überlebens auf 12,5 Monate erzielt werden [7]. Erst durch die Einführung einer Kombinationsbehandlung bestehend aus CYC und oralen Glukokorti- koiden (GC) wurde die Prognose dieser Patienten dramatisch verbessert. Durch dieses auch als Fauci-Schema bezeichnete Therapieregime konnten 75 bis 90\% der Patienten in Remission gebracht werden [8]. Es zeigte sich allerdings auch, dass es sich nicht um selbst-limitierte sondern um chronisch-rezidivierende Erkrankungen handelt, bei denen es innerhalb von fünf Jahren in 30 bis $50 \%$ der Patienten zu Rückfällen kommt [8]. Die deshalb in vielen Fällen notwendige andauernde oder erneute CYC-Gabe führte, bedingt durch dessen ausgeprägte Blasen- und Myelotoxizität sowie durch eine deutlich erhöhte Malignom- und Infektionsrate, zu einer erheblichen therapiebedingten Morbidität und Mortalität in bis zu 40\% der Patienten [9].

\section{Aktuelle Behandlungsstrategien
$\nabla$}

Die oben genannten Probleme des Fauci-Schemas führten dazu, dass ab Mitte der 90er-Jahre verstärkt differenzierende stadienund schweregradadaptierte Behandlungskonzepte in die Therapie der AASV eingeführt wurden, um hierdurch die Exposition mit CYC zu reduzieren [10]. Prinzipiell wird heutzutage zwischen der Behandlung einer floriden Erkrankungsphase mit dem Ziel der Remissionserreichung, der so genannten Induktionstherapie, und einer Erhaltungstherapie zur Rezidivprophylaxe bei Patienten in Remission unterschieden. Weiterhin wird bei der Induktionstherapie der Schwere- und Ausbreitungsgrad des vaskulitischen Organbefalls bzw. die Schwere der renalen Beteiligung berücksichtigt (siehe $\bullet$ Abb. 1).

Es werden hierbei lokalisierte bzw. früh-systemische Formen ohne unmittelbar bedrohliche Organmanifestationen von generalisierten Formen mit manifester oder drohender Organbeteiligung und schließlich den schwersten Verläufen mit Befall vitaler Organe unterschieden. Weitestgehend evidenz-basierte Therapieempfehlungen, die auf diesen Rationalen beruhen, sind inzwischen von internationalen medizinischen Fachgesellschaften veröffentlich worden [11,12]. Die wichtigsten Grundzüge stellen sich wie folgt dar, für eine schematische Übersicht siehe bitte - Abb. 1.

\section{Induktionsbehandlung \\ $\nabla$}

Das klassische Induktionsregime bei Patienten mit aktiver AASV und manifester bzw. unmittelbar bevorstehender relevanter Organbeteiligung (Glomerulonephritis, alveoläre Hämorrhagie, vaskulitische Neuropathie, etc.) besteht aus der Kombination von Hochdosis-Glukokortikoiden (GC, $1 \mathrm{mg} / \mathrm{kg}$ ) und CYC. In jüngster Zeit wird allerdings die kontinuierliche perorale CYCGabe $(2 \mathrm{mg} / \mathrm{kg})$ immer mehr durch die intermittierende i.v.Puls-Gabe $(15 \mathrm{mg} / \mathrm{kg})$ verdrängt $[11,12]$, da diese bei vergleichbarer Effektivität wegen der verringerten kumulativen CYCDosis wahrscheinlich besser verträglich ist [13]. Hierbei werden die ersten 3 Pulse alle 14 Tage gegeben, die folgenden in 3-4-wöchigen Abständen. Remissionsraten um 90\% sind mit beiden Methoden innerhalb von 6 Monaten möglich $[13,14]$. Bei schwerster Nierenbeteiligung, d.h. bereits bestehender oder kurz bevorstehender Dialysepflichtigkeit, sollte zusätzlich innerhalb der ersten 14 Behandlungstage ein 5-7-maliger Plasmaaustausch durchgeführt werden, da die renale Prognose hierdurch verbessert wird [15].

Bei Patienten mit weniger schweren Verläufen, d.h. ohne relevanten Nierenbefall und ohne unmittelbare Bedrohung vitaler 


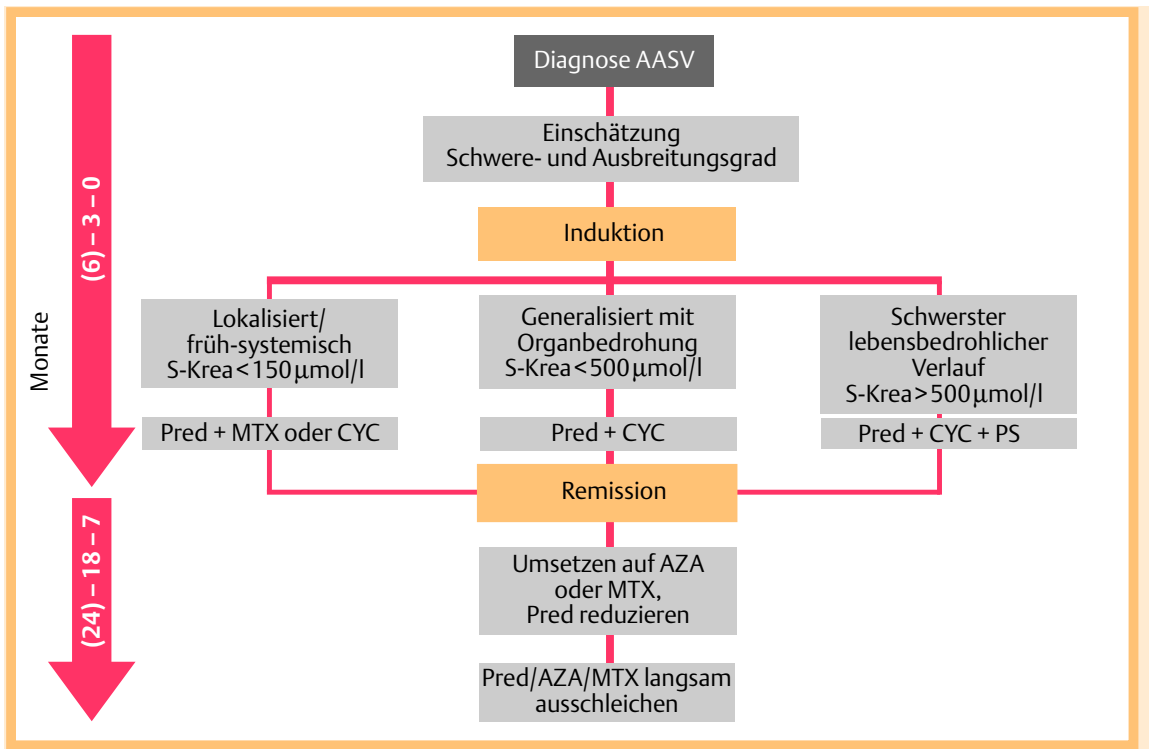

Abb. 1 Therapiealgorithmus AASV (modifiziert nach [12]). Abkürzungen: AASV, ANCA-assoziierte Vaskulitis; S-Krea, Serum-Kreatinin; Pred, Prednisolon; MTX, Methotrexat; CYC, Cyclophosphamid; PS, Plasmaseparation; AZA, Azathioprin.

Organfunktionen kann als Alternative eine Kombination von Hochdosis-GC und Methotrexat (MTX, 15-25 mg/Woche) durchgeführt werden. Hier liegen vor allem Erfahrungen bei Patienten mit WG und Befall der oberen und unteren Luftwege vor. Auch hier sind Remissionsraten um 90\% möglich. Es muss allerdings unter MTX von einer im Vergleich verlängerten Zeit bis zum Erreichen einer Remission ausgegangen werden [16].

Die Induktionsregimes werden immer in Kombination mit GC durchgeführt. Üblich sind initial Hochdosisgaben mit $1 \mathrm{mg} / \mathrm{kg}$ Prednisolon, wobei bei schwerster Organbeteiligung mit einer Methylprednisolonpulsbehandlung mit $1 \mathrm{gr} /$ die über 3 Tage begonnen werden kann. Die GC werden dann schrittweise innerhalb von 3 Monaten auf $0,25 \mathrm{mg} / \mathrm{kg}$ bzw. dann nach 6 Monaten auf 7,5-10 mg reduziert [17]. Vaskulitispatienten benötigen in der Regel eine längerfristige GC-Behandlung über der Cushingschwellendosis und benötigen deshalb eine medikamentöse Osteoporoseprophylaxe mit Vitamin D3 und Calcium bzw. beim Vorliegen besonderer Risikofaktoren zusätzlich Bisphosphonate. Während der Induktionsphase besteht für die Patienten eine erhöhte Infektgefahr insbesondere auch für opportunistische Erreger, der durch geeignete medikamentöse Prophylaxen begegnet werden muss. Bewährt haben sich als Pneumocystis jiroveci Prophylaxe die Gabe von Sulfamethoxazol/Trimethoprim (SMX/ TMP) $3 \times$ Woche 800/160 mg. Bei Patienten mit WG sollte SMX/ TMP unter Beachtung der Kontraindikationen in voller Dosierung von $2 \times 1$ täglich zusätzlich über den gesamten Behandlungszeitraum gegeben werden, weil hierdurch die Rezidivrate vor allem in HNO-Bereich signifikant gesenkt wird [18]. Bei allen CYC-enthaltenden Regimen, insbesondere bei i. v.-Gabe, muss an die beträchtliche Blasentoxizität des Medikamentes gedacht werden und eine entsprechende Prophylaxe mit MESNA erfolgen $[11,12]$.

\section{Remissionsbehandlung}

$\nabla$

Die Remissionsinduktion gelingt in der Regel bei der Mehrzahl der Patienten. Problematischer gestaltet sich häufig der Remissionserhalt. Grundsätzlich muss bei den AASV von Rezidiven in bis zu 50\% der Patienten innerhalb von 5 Jahren ausgegangen werden. Sichere Kriterien, die den individuellen Krankheitsver- lauf vorhersagen, sind nicht verfügbar. Als Remissionserhalt hat inzwischen das Azathioprin (AZA, $2 \mathrm{mg} / \mathrm{kg}$ ) das CYC verdrängt, da es wesentlich besser verträglich und bei Patienten in Remission zur Rezidivprophylaxe dem CYC gleichwertig ist [14]. Als Alternative bietet sich das MTX an, wobei hier eine eingeschränkte ( $\mathrm{S}$-Krea $>2 \mathrm{mg} / \mathrm{dl}$ ) oder eine instabile Nierenfunktion Kontraindikationen darstellen. Es muss ebenfalls unter MTX mit einer erhöhten Rezidivgefahr [14], vor allem auch in der Niere, gerechnet werden.

Bei Patienten mit Unverträglichkeiten auf die genannten Medikamente bieten sich als Alternativen in der Erhaltungstherapie die Immunsuppressiva Mycophenolat Mofetil [19] oder das Leflunomid [20] an, wobei für beide Medikamente nur kleine Kohorten untersucht sind.

Bezüglich der begleitenden CG-Behandlung hat sich bisher kein Standard etablieren können. Die diesbezüglich verfügbaren Studien befürworten eher eine längerfristige GC-Begleitbehandlung in Höhe von 7,5 mg/die Prednisolonäquivalent, da in allen Kohorten ohne GC bzw. mit frühem Absetzen eine gesteigerte Rezidivgefahr beobachtet werden konnte [17].

Die optimale Dauer der Erhaltungstherapie ist ebenfalls nicht gesichert. Auch hier legen die verfügbaren Daten eher einen längeren als einen zu kurzen Zeitraum nahe. Aktuelle Richtlinien empfehlen eine Behandlung über 18 bis 24 Monate nach Erreichen einer Vollremission mit einem remissonserhaltenden Regime, welches dann schrittweise reduziert wird [11,12].

\section{Besonderheiten des CSS $\nabla$}

Das CSS nimmt therapeutisch innerhalb der AASV eine Sonderstellung ein, da es besser auf eine Monotherapie mit GC anspricht. Zudem liegen im Vergleich zur WG oder MPA weniger randomisierte Studien zur Therapie vor. Grundsätzlich ist allerdings auch hier davon auszugehen, dass beim Vorliegen negativer prognostischer Prädiktoren (Herz-, Darm-, Nieren- oder ZNSBeteiligung) oder bei Rezidivneigung unter einer GC-Monotherapie eine frühzeitige Kombination mit CYC in Analogie zur WG oder MPA unter Verwendung des i.v.-Bolus-Regimes vorteilhaft ist [21]. 


\section{Experimentelle Therapieansätze \\ $\nabla$}

Bei Patienten mit therapierefraktärem Verlauf wurden in Studien oder als Heilversuch bereits verschiedene andere immunsuppressive Konzepte erfolgreich eingesetzt. Zum einen war die T-Zell-depletierende Therapie mit Antithymocytenglobulin (ATG) erfolgreich [22]. ATG enthält polyklonale anti-T-Zell-Antikörper und führt so zum T-Zell-Zerfall; es wurde gut toleriert, wenn zuvor simultane Infektionen ausgeschlossen wurden. Ein anderer in einer kleinen Studie erfolgreicher Therapieansatz bei therapierefraktären Patienten ist die zyklische Gabe von 15-Deoxyspergualin (DSG), ein synthetisches Spergualin-Analog, das zunächst in der Transplantationsimmunologie eingesetzt wurde [23]. Eine andere vielversprechende therapeutische Alternative bei Patienten, die nicht hinreichend auf Cyclophosphamid und Steroide ansprechen, bietet die B-Zell-Depletion mit Rituximab. Rituximab ist ein anti-CD20-Antikörper, der zu einer Depletion der CD20-positiven B-Zellen und zum Rückgang der ANCA-Titer führt, ohne jedoch eine generalisierte Immunsupression zu verursachen. In mehreren kleineren Studien und Fallberichten wurde Rituximab erfolgreich zur Remmisionsinduktion eingesetzt [24].

\section{Fazit für die Praxis \\ $\nabla$}

AASV müssen trotz ihrer relativen Seltenheit grundsätzlich in die Differenzialdiagnose unklarer systemischer Krankheitszustände, insbesondere wenn sie entzündlicher Natur sind, einbezogen werden. ANCA-Bestimmungen zählen heute zu den Standardbestimmungen in der Differentialdiagnostik vaskulitischer Erkrankungen und haben die Diagnose der AASV wesentlich erleichtert. Die chronisch-rezidivierend verlaufenden AASV benötigen eine stadien- und schweregradadaptierte immunsuppressive Therapie, die inzwischen in weiten Teilen evidenzbasiert sind und eine verbesserte und differenzierte Behandlung dieser Erkrankungen ermöglichen.

\section{Abstract}

\section{ANCA-Associated Vasculitides: Current Therapeutic Trends} $\nabla$

ANCA-associated vasculitides (AASV) belong to the group of the primary systemic vasculitides and predominantly affect small und medium-sized vessels. Pathohistologically, they are characterized by a necrotizing pauci-immune angiitis. Circulating disease-specific anti-neutrophil cytoplasmic antibodies (ANCA) can be found in the majority of these patients. AASV are clinically heterogeneous diseases which, after a common unspecific prodromal phase, lead to an overt vasculitic disease. By nature, virtually each organ may be affected, though a certain preponderance for kidney and upper and lower respiratory tract involvement is observed. Skin involvement is also found in about $50 \%$ of affected patients. Untreated, AASV have a dismal prognosis, but current stage and severity adapted immunosuppressive regimens lead to a $90 \%$ remission rate. Due to the chronic relapsing nature of AASV, maintenance therapy is also necessary. Due to complex treatment schemes as well as the unpredictable course of these diseases patients with AASV should be treated and followed in experienced vasculitis centers.

\section{Literatur}

1 Falk RJ, Jennette JC. ANCA small-vessel vasculitis. J Am Soc Nephrol 1997; 8: $314-322$

2 Jennette JC, Falk RJ, Andrassy K et al. Nomenclature of systemic vasculitides. Proposal of an international consensus conference. Arthritis Rheum 1994; 37: 187-192

3 Jennette JC, Falk RJ. Small-vessel vasculitis. N Engl J Med 1997; 337: $1512-1523$

4 Birck R, Van Der Woude FJ. Rapidly progressive glomerulonephritis:classification, pathogenesis and clinical management. Internist (Berl) 2003; 44: 1107-1119

5 Brons RH, de Jong MC, de Boer NK et al. Detection of immune deposits in skin lesions of patients with Wegener's granulomatosis. Ann Rheum Dis 2001; 60: 1097-1102

6 Carlson JA, Chen KR. Cutaneous vasculitis update: small vessel neutrophilic vasculitis syndromes. Am J Dermatopathol 2006; 28: 486 - 506

7 Walton EW. Giant-cell granuloma of the respiratory tract (Wegener's granulomatosis). Br Med J 1958; 2: $265-270$

8 Hoffman GS, Kerr GS, Leavitt RY et al. Wegener granulomatosis: an analysis of 158 patients. Ann Intern Med 1992; 116: 488 - 498

9 Langford CA. Management of systemic vasculitis. Best Pract Res Clin Rheumatol 2001; 15: 281 - 297

10 Jayne $D R$, Rasmussen $N$. Treatment of antineutrophil cytoplasm autoantibody-associated systemic vasculitis: initiatives of the European Community Systemic Vasculitis Clinical Trials Study Group. Mayo Clin Proc 1997; 72: 737- 747

11 Mukhtyar C, Guillevin L, Cid MC et al. EULAR Recommendations for the management of primary small and medium vessel vasculitis. Ann Rheum Dis 2008; April 15: Epub ahead of print

12 Lapraik C, Watts R, Bacon P et al. BSR and BHPR guidelines for the management of adults with ANCA associated vasculitis. Rheumatology (Oxford) 2007; 46: 1615-1616

13 de Groot K, Jayne DR, Tesar V, Savage CO. European, multicenter randomised controlled trial of daily oral versus pulse cyclophosphamide for induction of remission in ANCA-associated systemic vasculitis for the European Vasculitis Study Group. J Am Soc Nephrol 2005; 7A

14 Jayne $D$, Rasmussen $N$, Andrassy $K$ et al. A randomized trial of maintenance therapy for vasculitis associated with antineutrophil cytoplasmic autoantibodies. N Engl J Med 2003; 349: 36 - 44

15 Jayne DR, Gaskin G, Rasmussen N et al. Randomized trial of plasma exchange or high-dosage methylprednisolone as adjunctive therapy for severe renal vasculitis. J Am Soc Nephrol 2007; 18: 2180-2188

16 De Groot K, Rasmussen N, Bacon PA et al. Randomized trial of cyclophosphamide versus methotrexate for induction of remission in early systemic antineutrophil cytoplasmic antibody-associated vasculitis. Arthritis Rheum 2005; 52: 2461 - 2469

17 Goek ON, Stone JH. Randomized controlled trials in vasculitis associated with anti-neutrophil cytoplasmic antibodies. Curr Opin Rheumatol 2005; 17: 257-264

18 Stegeman CA, Tervaert JW, de Jong PE, Kallenberg CG. Trimethoprimsulfamethoxazole (co-trimoxazole) for the prevention of relapses of Wegener's granulomatosis. Dutch Co-Trimoxazole Wegener Study Group. N Engl J Med 1996; 335: 16-20

19 Koukoulaki M, Jayne DR. Mycophenolate mofetil in anti-neutrophil cytoplasm antibodies-associated systemic vasculitis. Nephron Clin Pract 2006; 102: $\mathrm{c} 100-\mathrm{c} 107$

20 Metzler C, Miehle N, Manger $K$ et al. Elevated relapse rate under oral methotrexate versus leflunomide for maintenance of remission in Wegener's granulomatosis. Rheumatology (Oxford) 2007; 46: 1087 1091

21 Pagnoux C, Guilpain P, Guillevin L. Churg-Strauss syndrome. Curr Opin Rheumatol 2007; 19: 25-32

22 Schmitt WH, Hagen EC, Neumann I et al. Treatment of refractory Wegener's granulomatosis with antithymocyte globulin (ATG): an open study in 15 patients. Kidney Int 2004; 65: 1440-1448

23 Birck R, Warnatz K, Lorenz HM et al. 15-Deoxyspergualin in patients with refractory ANCA-associated systemic vasculitis: a six-month open-label trial to evaluate safety and efficacy. J Am Soc Nephrol 2003; 14: 440-447

24 Walsh M, Jayne D. Rituximab in the treatment of anti-neutrophil cytoplasm antibody associated vasculitis and systemic lupus erythematosus: past, present and future. Kidney Int 2007; 72: 676-682 